\title{
SYLVESTER-BASED PRECONDITIONING FOR THE WAVEGUIDE EIGENVALUE PROBLEM
}

\author{
EMIL RINGH*, GIAMPAOLO MELE*, JOHAN KARLSSON*, ELIAS JARLEBRING*
}

\begin{abstract}
We consider a nonlinear eigenvalue problem (NEP) arising from absorbing boundary conditions in the study of a partial differential equation (PDE) describing a waveguide. We propose a new computational approach for this large scale NEP based on residual inverse iteration (Resinv) with preconditioned iterative solves. Similar to many preconditioned iterative methods for discretized PDEs, this approach requires the construction of an accurate and efficient preconditioner. For the waveguide eigenvalue problem, the associated linear system can be formulated as a generalized Sylvester equation. The equation is approximated by a low-rank correction of a Sylvester equation, which we use as a preconditioner. The action of the preconditioner is efficiently computed using the matrix equation version of the Sherman-Morrison-Woodbury (SMW) formula. We show how the preconditioner can be integrated into Resinv. The results are illustrated by applying the method to large-scale problems.
\end{abstract}

1. Introduction. We are concerned with the study of propagation of waves in a waveguide. The application of two well established techniques (Floquet theory and absorbing boundary conditions) leads to the following characterization of wave propagation in $\mathbb{R}^{2}$. Details of such a derivation can be found, e.g., in [15, 34].

The characterization is described by a PDE on a rectangular domain $S_{0}=$ $\left[x_{-}, x_{+}\right] \times[0,1]$. More precisely, we wish to compute $u: S_{0} \rightarrow \mathbb{C}$ and $\gamma \in \mathbb{C}$ such that

$$
\begin{aligned}
\Delta u(x, z)+2 \gamma u_{z}(x, z)+\left(\gamma^{2}+\kappa^{2}(x, z)\right) u(x, z) & =0 & & (x, z) \in S_{0} \\
u(x, 0) & =u(x, 1) & & x \in\left(x_{-}, x_{+}\right) \\
u_{z}(x, 0) & =u_{z}(x, 1) & & x \in\left(x_{-}, x_{+}\right) \\
\mathcal{T}_{-, \gamma}\left[u\left(x_{-}, \cdot\right)\right](z) & =-u_{x}\left(x_{-}, z\right) & & z \in(0,1) \\
\mathcal{T}_{+, \gamma}\left[u\left(x_{+}, \cdot\right)\right](z) & =u_{x}\left(x_{+}, z\right) & & z \in(0,1) .
\end{aligned}
$$

The operators $\mathcal{T}_{-, \gamma}$ and $\mathcal{T}_{+, \gamma}$ are the so-called Dirichlet-to-Neumann (DtN) maps, which we specify in Section 2. The spatially dependent constant $\kappa(x, z)$ is the wavenumber, which in our work is assumed to be piecewise constant. A benchmark example is illustrated in Figure 1.1.

Note that (1.1) is a PDE-eigenvalue problem, where the eigenvalue $\gamma$ appears in a nonlinear way in the operator as well as in the boundary conditions, due to the $\gamma$-dependence of the DtNs. The problem (1.1) will be referred to as the waveguide eigenvalue problem (WEP) and we discretize this PDE in a way that allows us to construct an efficient iterative procedure. More precisely, we derive results and methods with a uniform finite-difference (FD) discretization, and also investigate its use in combination with a finite-element method (FEM) discretization. The discretization is presented in Section 2.2. Due to the nonlinearity in the PDE-eigenvalue problem, the discretized problem is a nonlinear eigenvalue problem (NEP) of the form: find $(\gamma, v) \in \mathbb{C} \times \mathbb{C}^{n_{z} n_{x}+2 n_{z}} \backslash\{0\}$ such that

$$
M(\gamma) v=0,
$$

where $n_{x}$ and $n_{z}$ are the number of discretization points in $x$ - and $z$-direction respectively.

*Dept. Mathematics, KTH Royal Institute of Technology, Lindstedtsvgen 25, Stockholm, Sweden, email: \{eringh, gmele, eliasj\}@kth.se, johan.karlsson@math.kth.se 


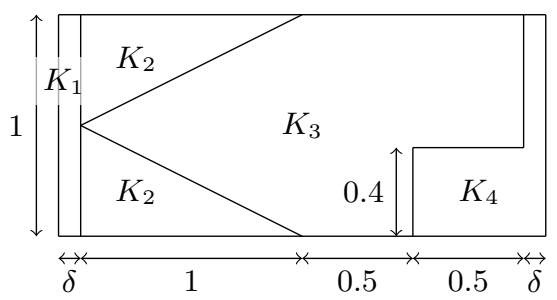

(a) Geometry of a benchmark waveguide.

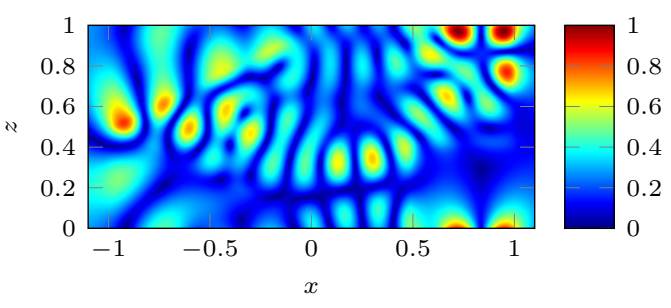

(b) The absolute value of an eigenfunction.

FIGURE 1.1. Geometry of the benchmark waveguide and an eigenfunction corresponding to the eigenvalue $\gamma \approx-1.341-1.861 i$. The same waveguide is used in the numerical examples, Section 6. The values $K_{i}$ indicates regions where the wavenumber $\kappa(x, z)$ is constant. For this waveguide $K_{1}=\sqrt{2.3} \pi, K_{2}=2 \sqrt{3} \pi, K_{3}=4 \sqrt{3} \pi, K_{4}=\pi$, and $\delta=0.1$.

Over the last decades, the NEP has been considerably studied in the numerical linear algebra community, and there is a large family of different numerical methods, which we briefly summarize as follows. A number of methods can be seen as flavors of Newton's method, e.g., block Newton methods [18], generalizations of inverse iteration $[22,26]$ and generalizations of the Jacobi-Davidson method. See PhD thesis [29] for a summary of these methods. A number of approaches are based on numerically computing a contour integral $[1,6]$, which can be accelerated as described in [38] and references therein. Krylov methods and rational Krylov methods have been generalized in various ways, e.g., the Arnoldi based methods [36, 17], rational Krylov approaches $[12,3,4]$. See the summary papers $[21,26,37]$ and the benchmark collection [5] for further literature on methods for nonlinear eigenvalue problems.

Most of these methods involve the solution to the associated linear system of equations

$$
M(\sigma) y=r
$$

For large-scale problems, the solution to this linear system is often restricting the applicability of the method. In this work we adapt the method called residual inverse iteration (Resinv) which was developed in [22]. Resinv is an iterative method for computing the eigenvalue closest to a given shift $\sigma \in \mathbb{C}$ and it has the attractive feature that the shift $\sigma$ is kept constant throughout the iterations.

The constant shift allows for precomputation, which reduces the computational effort for solving the linear systems (1.3). The standard way to exploit this is to pre-compute the LU-factorization of $M(\sigma)$. Unfortunately this is not effective for our large-scale problem, due to memory requirements. Instead, we propose to solve (1.3) with a preconditioned iterative method such as GMRES [28] or BiCGStab [35], where the constant shift and the structure of $M(\sigma)$ allows us to carry out substantial precomputations in the initialization of Resinv.

A number of recent approaches exploit that a uniform discretization of a rectangular domain PDE can be expressed as a matrix equation, e.g., using the Sylvester equations or Lyapunov equations. The matrix-equation approach has been used, e.g., in the setting of convection-diffusion equations [23], fractional differential equations [32], PDE-constrained optimization [7], and stochastic differential equations [24]. Inspired by this, we propose a new preconditioner for the WEP based on matrix equations. As a first step, shown in Proposition 2.1 and Section 3, the linear system of 
equations (1.3) is formulated as a matrix equation. In Section 4, this matrix equation is approximated by a low-rank correction of a Sylvester equation, i.e., of the form

$$
\mathcal{L}(X)+\Pi(X)=C
$$

where $\mathcal{L}$ is a Sylvester operator and $\Pi$ is a low-rank linear operator of the form $\Pi(X):=\sum_{k=1}^{N} \mathscr{W}_{k}(X) E_{k}$, and where $\mathscr{W}_{k}: \mathbb{C}^{n \times n} \rightarrow \mathbb{C}, k=1, \ldots, N$ are linear functionals. We use a matrix equation version of the Sherman-Morrison-Woodbury (SMW) formula to solve (1.4). In Section 5 we describe how this can be done in a fast and memory efficient manner using the structures in our problem. A dominating part of the computation is independent of $C$ and can therefore be precomputed. Properties of the approach are illustrated in Section 6, where we also compare the performance with other approaches.

The following notation is adopted in this paper. We let $A \circ B$ denote the Hadamard, or element-wise, matrix product between $A$ and $B$, and $A \otimes B$ denotes the Kronecker product. We let $\operatorname{vec}(A) \in \mathbb{C}^{n m}$ denote the vectorization of $A \in \mathbb{C}^{n \times m}$, i.e., the vector obtained by stacking the columns of $A$ on top of each other. The set of eigenvalues of the matrix $A$ is denoted $\operatorname{eig}(A)$. The $n \times n$ identity matrix is denoted $I_{n}$. The the matrix $J_{n} \in \mathbb{R}^{n \times n}$ denotes the flipped identity matrix, that is $\left[J_{n}\right]_{k, \ell}=1$ if $k=n-\ell+1$ and 0 otherwise. The column vector consisting of ones is denoted by 1 .

\section{Background and preliminaries.}

2.1. Problem background. The PDE (1.1) stems from the propagation of waves in a periodic medium. The derivation can be briefly summarized as follows. See $[15,34]$ for details.

Consider Helmholtz's equation

$$
\Delta v(x, z)+\kappa(x, z)^{2} v(x, z)=0 \quad(x, z) \in \mathbb{R}^{2},
$$

where $\kappa(x, z) \in L^{\infty}\left(\mathbb{R}^{2}\right)$ is the wavenumber. The wavenumber is a 1-periodic function in the $z$-direction which is constant for sufficiently large $|x|$, i.e., $\kappa(x, z+1)=\kappa(x, z)$ for all $x, z$, and there exists real numbers $\xi_{-}$and $\xi_{+}$such that $\kappa(x, z)=\kappa_{-}$for $x<\xi_{-}$ and $\kappa(x, z)=\kappa_{+}$for $x>\xi_{+}$. We use the ansatz

$$
v(x, z)=e^{\gamma z} u(x, z)
$$

where $u(x, z+1)=u(x, z)$ in (2.1) and we apply absorbing boundary conditions at $x=x_{-} \leq \xi_{-}$and $x=x_{+} \geq \xi_{+}$. From this ansatz we directly identify that $u$ satisfies (1.1a). A more precise analysis (presented in [15]) shows that also (1.1d)-(1.1e) are satisfied where the DtN-maps are defined by

$$
\mathcal{T}_{ \pm, \gamma}[g](z):=\sum_{k \in \mathbb{Z}} s_{ \pm, k}(\gamma) g_{k} e^{2 \pi i k z}
$$

where $\left\{g_{k}\right\}_{k \in \mathbb{Z}}$ are the Fourier series coefficients of the function $g(z)$ and $s_{k}, k \in \mathbb{Z}$, are given by

$$
\begin{aligned}
& s_{ \pm, k}(\gamma):=\operatorname{sign}\left(\operatorname{Im}\left(\beta_{ \pm, k}(\gamma)\right)\right) i \sqrt{\beta_{ \pm, k}(\gamma)} \\
& \beta_{ \pm, k}(\gamma):=(\gamma+2 \pi i k)^{2}+\kappa_{ \pm}^{2} .
\end{aligned}
$$


2.2. Discretization of the WEP. The PDE (1.1) is in this work discretized as follows. We use a uniform FD discretization with $n_{x}$ and $n_{z}$ points in $x$ - and $z$ direction respectively. The grid consists of the points $x_{k}=x_{-}+k h_{x}$ for $k=1,2, \ldots n_{x}$ where $h_{x}=\left(x_{+}-x_{-}\right) /\left(n_{x}+1\right)$, and $z_{\ell}=\ell h_{z}$ for $\ell=1,2, \ldots, n_{z}$ where $h_{z}=1 / n_{z}$.

This FD-discretization leads to the NEP (1.2) being described by the following block matrix

$$
M(\gamma):=\left(\begin{array}{cc}
Q(\gamma) & C_{1} \\
C_{2}^{T} & P(\gamma)
\end{array}\right)
$$

Here $Q(\gamma)$ represents the discretization of the interior (1.1a) and $P(\gamma)$ represents the Dirichlet-to-Neumann maps, (1.1d) and (1.1e). The matrix $C_{1}$ represents the effect of the boundary points to the interior and $C_{2}^{T}$ represents the effect of the interior on the boundary constraints, i.e., (1.1d) and (1.1e). The matrix $Q(\gamma) \in \mathbb{C}^{n_{x} n_{z} \times n_{x} n_{z}}$ is large and sparse, and given by

$$
Q(\gamma):=A_{0}+\gamma A_{1}+\gamma^{2} A_{2}
$$

with $A_{0}:=D_{x x}^{T} \otimes I_{n_{z}}+I_{n_{x}} \otimes D_{z z}+\operatorname{diag}(\operatorname{vec}(K))$, and $A_{1}:=2 I_{n_{x}} \otimes D_{z}$, and $A_{2}:=$ $I_{n_{x} n_{z}}$. Here $D_{x x} \in \mathbb{R}^{n_{x} \times n_{x}}$ is the second derivative matrix, and $D_{z}, D_{z z} \in \mathbb{R}^{n_{z} \times n_{z}}$ are the circulant first and second derivative matrices. That is, $D_{x x}=\left(-2 I_{n_{x}}+Z_{n_{x}}+\right.$ $\left.Z_{n_{x}}^{T}\right) / h_{x}^{2}, D_{z}=\left(Z_{n_{z}}+e_{1} e_{n_{z}}^{T}-Z_{n_{z}}^{T}-e_{n_{z}} e_{1}^{T}\right) /\left(2 h_{z}\right)$, and $D_{z z}=\left(-2 I_{n_{z}}+Z_{n_{z}}+e_{1} e_{n_{z}}^{T}+\right.$ $\left.Z_{n_{z}}^{T}+e_{n_{z}} e_{1}^{T}\right) / h_{z}^{2}$, where $Z_{n} \in \mathbb{R}^{n \times n}$ is the shift matrix, defined by $\left[Z_{n}\right]_{k, l}=1$ if $k-l=1$ and 0 otherwise.

The matrix $K$ is the discretization of the squared wavenumber, i.e., $[K]_{k, \ell}:=$ $\kappa^{2}\left(x_{\ell}, z_{k}\right)$. The block $C_{1} \in \mathbb{C}^{n_{x} n_{z} \times 2 n_{z}}$ is given by

$$
C_{1}:=\frac{1}{h_{x}^{2}}\left(e_{1} \otimes I_{n_{z}}, e_{n_{x}} \otimes I_{n_{z}}\right)
$$

and the block $C_{2}^{T} \in \mathbb{C}^{2 n_{z} \times n_{x} n_{z}}$ is given by

$$
C_{2}^{T}:=\left(\begin{array}{c}
d_{1} e_{1}^{T} \otimes I_{n_{z}}+d_{2} e_{2}^{T} \otimes I_{n_{z}} \\
d_{1} e_{n_{x}}^{T} \otimes I_{n_{z}}+d_{2} e_{n_{x}-1}^{T} \otimes I_{n_{z}}
\end{array}\right),
$$

with $d_{1}:=\frac{2}{h_{x}} d_{2}:=-\frac{1}{2 h_{x}}$. The last block $P(\gamma) \in \mathbb{C}^{2 n_{z} \times 2 n_{z}}$ stems from the discretization of the DtN operators, i.e., the operators in the left hand side of (1.1d) and (1.1e). We truncate the Fourier series expansion in (2.2) and use only the coefficients corresponding to $-p, \ldots, p$, we choose $p$ such that $n_{z}=2 p+1$. Then

$$
P(\gamma):=\left(\begin{array}{cc}
P_{-}(\gamma) & 0 \\
0 & P_{+}(\gamma)
\end{array}\right)=\left(\begin{array}{cc}
R \Lambda_{-}(\gamma) R^{-1} & 0 \\
0 & R \Lambda_{+}(\gamma) R^{-1}
\end{array}\right)
$$

with $\Lambda_{ \pm}:=\operatorname{diag}\left(S_{ \pm}\right)$, where $S_{ \pm}:=\left(s_{ \pm,-p}+d_{0}, s_{ \pm,-p+1}+d_{0}, \ldots, s_{ \pm, p}+d_{0}\right)$, and $d_{0}:=-\frac{3}{2 h_{x}}$, and where $s_{ \pm, k}$ are defined by (2.3a). Moreover, $[R]_{k, \ell}=e^{2 \pi i(\ell-p-1) k h_{z}}$ and $R^{*}=n_{z} R^{-1}$.

REMARK 1. Note that $R$ is the Fourier matrix left multiplied with the antidiagonal matrix with $\left(1, e^{2 \pi i p h_{z}}, e^{2 \pi i 2 p h_{z}}, \ldots, e^{2 \pi\left(n_{z}-1\right) p h_{z}}\right)$ on its anti-diagonal. Consequently, the action of both $R$ and $R^{-1}$ on a vector can be efficiently calculated with the Fast Fourier Transform (FFT) and from (2.8) we conclude that calculating the action of $P_{-}(\gamma), P_{-}(\gamma)^{-1}, P_{+}(\gamma)$, and $P_{+}(\gamma)^{-1}$ on a vector can be done in $\mathcal{O}\left(n_{z} \log \left(n_{z}\right)\right)$ operations. 
For future reference, we now also note that when $\gamma$ is in the left half-plane of $\mathbb{C}$ the derivative of $M(\gamma)$ with respect to $\gamma$ is given by

$$
M^{\prime}(\gamma):=\left(\begin{array}{cc}
Q^{\prime}(\gamma) & 0 \\
0 & P^{\prime}(\gamma)
\end{array}\right)
$$

where $Q^{\prime}(\gamma):=A_{1}+2 \gamma A_{2}$ and $P^{\prime}(\gamma)=\operatorname{diag}\left(R \Lambda_{-}^{\prime}(\gamma) R^{-1}, R \Lambda_{+}^{\prime}(\gamma) R^{-1}\right)$. The matrices are directly given by $\Lambda_{ \pm}^{\prime}:=\operatorname{diag}\left(S_{ \pm}^{\prime}\right)$, where $S_{ \pm}^{\prime}:=\left(s_{ \pm,-p}^{\prime}, s_{ \pm,-p+1}^{\prime}, \ldots, s_{ \pm, p}^{\prime}\right)$, $s_{ \pm, k}^{\prime}(\gamma):=\operatorname{sign}\left(\operatorname{Im}\left(\beta_{ \pm, k}(\gamma)\right)\right) i(\gamma+2 \pi i k) / \sqrt{\beta_{ \pm, k}(\gamma)}$, and $\beta_{ \pm, k}(\gamma)$ are given by $(2.3 \mathrm{~b})$.

2.3. Residual inverse iteration for the WEP. Our approach is based on the Resinv [22] as a solution method for the NEP (1.2) with $M$ defined by (2.4). Given an approximation to the eigenpair $\left(\gamma_{k}, v_{k}\right)$, Resinv iteratively computes new approximations in each iteration. The procedure consists of a few steps. The first step is to compute a new approximation of the eigenvalue $\gamma_{k+1}$ by solving the nonlinear scalar equation

$$
v_{k}^{*} M\left(\gamma_{k+1}\right) v_{k}=0
$$

There are different ways of choosing the left vector in (2.10) discussed in the literature $[22,16,29]$, but we choose the current approximation of the right eigenvector, as it is presented in the equation. Equation (2.10) is solved with Newton's method in one unknown variable which requires that we calculate the derivative of $v_{k}^{*} M(\gamma) v_{k}$ with respect to $\gamma$. The derivative, for $\gamma$ in the left half-plane of $\mathbb{C}$, can be computed from (2.9). The second step consists of computing a residual

$$
r_{k}=M\left(\gamma_{k+1}\right) v_{k}
$$

Subsequently $r_{k}$ is used to calculate a correction to the eigenvector by solving

$$
\Delta v_{k}=M(\sigma)^{-1} r_{k}
$$

where $\sigma$ is a fixed shift that is used throughout the whole procedure. We propose to use a preconditioned iterative method to solve (2.12). The third and last step is to update the eigenvector approximation $v_{k+1}=v_{k}-\Delta v_{k}$, and to normalize it $v_{k+1}=v_{k+1} /\left\|v_{k+1}\right\|$. The Resinv procedure is summarized in Algorithm 1 .

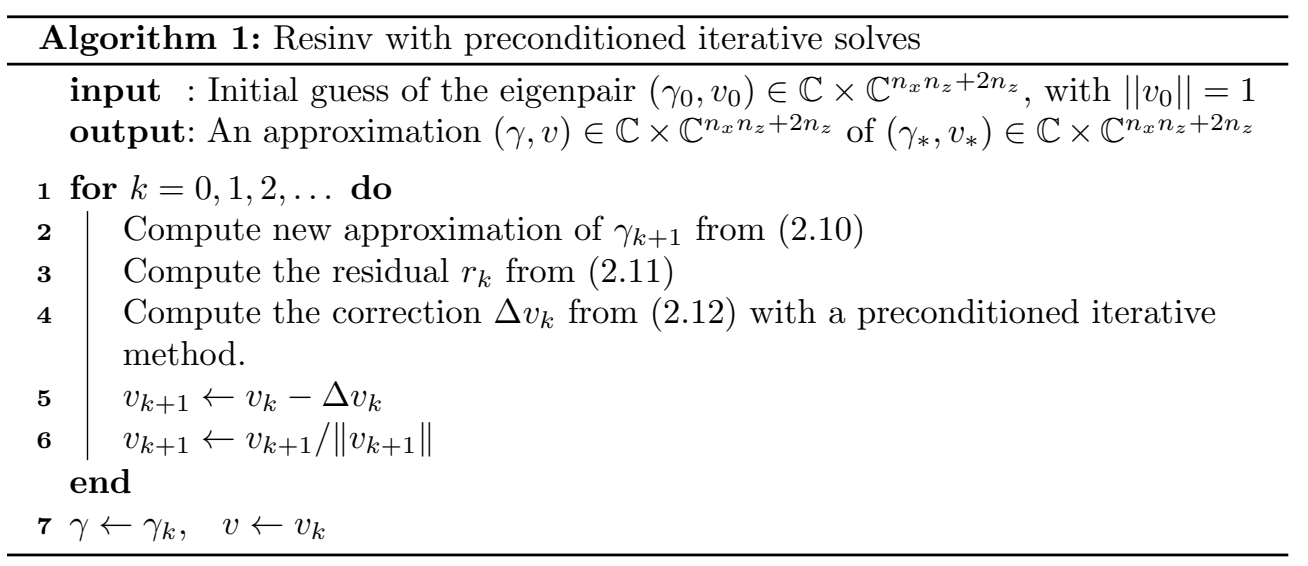


Large parts of the computational effort in Algorithm 1 often consists of the solving of the linear system (2.12) and we present a method that makes the computation feasible for large-scale problems. We use the Schur complement of $M(\sigma)$ with respect to the block $P(\sigma)$,

$$
S(\sigma):=Q(\sigma)-C_{1} P(\sigma)^{-1} C_{2}^{T},
$$

to specialize the computation of (2.12) for the WEP. The specialization is an important step in our algorithm and therefore we present it in the following form.

Proposition 2.1 (Schur complement for the WEP). Let $M(\sigma)$ be as in (2.4), the shift $\sigma \in \mathbb{C}$, and let $S(\sigma)$ be the Schur complement (2.13). Moreover, let $r \in$ $\mathbb{C}^{n_{x} n_{z}+2 n_{z}}$, and let $r_{\text {int }}$ be the first $n_{x} n_{z}$ elements of $r$ and $r_{\text {ext }}$ be the last $2 n_{z}$ elements of $r$. Then

$$
M(\sigma)^{-1} r=\left(\begin{array}{c}
q \\
P(\sigma)^{-1}\left(-C_{2}^{T} q+r_{e x t}\right)
\end{array}\right)
$$

where

$$
q:=S(\sigma)^{-1} \tilde{r}
$$

and

$$
\tilde{r}:=r_{\text {int }}-C_{1} P(\sigma)^{-1} r_{\text {ext }}
$$

We use Proposition 2.1 to solve the linear system in Step 4 in Algorithm 1. More precisely, we use a preconditioned iterivative method to solve (2.15), and FFT to compute the action $P(\sigma)^{-1}$ (as described in Remark 1). All other operations required for the application proposition have negligable computational cost.

3. Matrix equation characterization. In order to construct a good preconditioner for the linear system (2.15) we now formulate it as a matrix equation. Without loss of generality we express $(2.15)$ as $S(\sigma) \operatorname{vec}(X)=\operatorname{vec}(C)$, where $X, C \in \mathbb{C}^{n_{z} \times n_{x}}$.

Note that $S(\sigma)$ is defined in (2.13) as the sum of $Q(\sigma)$ and $-C_{1} P(\sigma)^{-1} C_{2}^{T}$, where $Q(\sigma)$ is described by (2.5). The action of $Q(\sigma)$ can be characterized with matrix equations. By direct application of rules for Kronecker products, see e.g., [14, Section 4.3], it follows that

$$
Q(\sigma) \operatorname{vec}(X)=\operatorname{vec}\left(\left(D_{z z}+2 \sigma D_{z}+\sigma^{2} I_{n_{z}}\right) X+X D_{x x}+K \circ X\right) .
$$

The action of the first two terms of (3.1) can be identified with a Sylvester operator, $\mathscr{L}: \mathbb{C}^{n_{z} \times n_{x}} \rightarrow \mathbb{C}^{n_{z} \times n_{x}}:$

$$
\mathscr{L}(X):=A X+X B,
$$

and hence the action of $Q(\sigma)$ can be viewed as a generalized Sylvester operator. The action corresponding to $S(\sigma)$ can similarly also be constructed as a generalization of the Sylvester operator. We formalize it in the following result, where we also introduce an additional free parameter $\bar{k}$. This parameter is later chosen in such a way that the contribution of the terms corresponding to the Sylvester operator is large.

Proposition 3.1 (Waveguide matrix equation). Let $X \in \mathbb{C}^{n_{z} \times n_{x}}$, let $C \in$ $\mathbb{C}^{n_{z} \times n_{x}}$ be a given matrix, and let $S(\sigma)$ be the Schur complement $(2.13)$. Then $\operatorname{vec}(X)$ is a solution to $S(\sigma) \operatorname{vec}(X)=\operatorname{vec}(C)$ if and only if $X$ is a solution to

$$
A X+X B+\left(K-\bar{k} \mathbf{1 1}{ }^{T}\right) \circ X-P_{-}(\sigma)^{-1} X E-P_{+}(\sigma)^{-1} X J_{n_{x}} E J_{n_{x}}=C,
$$


where $A:=D_{z z}+2 \sigma D_{z}+\sigma^{2} I_{n_{z}}+\bar{k} I_{n_{z}}$, and $B:=D_{x x}$, and $E:=\frac{1}{h_{x}^{2}}\left(d_{1} e_{1}+d_{2} e_{2}\right) e_{1}^{T}$, $d_{1}$ and $d_{2}$ is given by the discretization, $J_{n_{x}}$ is the flipped identity, and $\bar{k}$ is a free parameter.

Proof. We have that $S(\sigma)=Q(\sigma)-C_{1} P(\sigma)^{-1} C_{2}^{T}$. The equivalent matrix equation formulation for $Q(\sigma)$ is found apparent from (3.1). The rest follows from the calculation

$$
\begin{aligned}
& C_{1} P(\sigma)^{-1} C_{2}^{T} \\
& =\frac{1}{h_{x}^{2}}\left(e_{1} \otimes I_{n_{z}}, e_{n_{x}} \otimes I_{n_{z}}\right)\left(\begin{array}{cc}
P_{-}(\sigma)^{-1} & 0 \\
0 & P_{+}(\sigma)^{-1}
\end{array}\right)\left(\begin{array}{c}
d_{1} e_{1}^{T} \otimes I_{n_{z}}+d_{2} e_{2}^{T} \otimes I_{n_{z}} \\
d_{1} e_{n_{x}}^{T} \otimes I_{n_{z}}+d_{2} e_{n_{x}-1}^{T} \otimes I_{n_{z}}
\end{array}\right) \\
& =e_{1}\left(\frac{d_{1}}{h_{x}^{2}} e_{1}^{T}+\frac{d_{2}}{h_{x}^{2}} e_{2}^{T}\right) \otimes P_{-}(\sigma)^{-1}+e_{n_{x}}\left(\frac{d_{1}}{h_{x}^{2}} e_{n_{x}}^{T}+\frac{d_{2}}{h_{x}^{2}} e_{n_{x}-1}^{T}\right) \otimes P_{+}(\sigma)^{-1} .
\end{aligned}
$$

$\square$

\section{The Sylvester SMW structure for the WEP.}

4.1. Sylvester-type SMW-structure. Our computational procedure is based on the explicit formula for the inverse of a matrix with a low-rank correction, the Sherman-Morrison-Woodbury (SMW) formula [11, Equation (2.1.4)]. We use the formulation

$$
\left(L+U V^{T}\right)^{-1} c=L^{-1}\left(c-U W^{-1} V^{T} L^{-1} c\right)
$$

where $U, V \in \mathbb{C}^{n \times N}$ and

$$
W:=I+V^{T} L^{-1} U \in \mathbb{C}^{N \times N} .
$$

In order to apply the SMW-formula to equations of the form (3.3), we need a particular matrix equation version of the SMW-formula. The adaption of SMW-formula's to matrix equations has been examined previously in the literature $[8,19,25]$. Our formulation is based on a specialization of [8, Lemma 3.1] that is set up to minimize the memory requirements (as we further discuss in Remark 2).

We select the $L$-matrix in (4.1) as the vectorization of a Sylvester operator (3.2), which is invertible if $\operatorname{eig}(A) \cap \operatorname{eig}(-B)=\emptyset$, see e.g., [14, Theorem 4.4.6]. We make this specific choice since the solution to the Sylvester equation in our case be computed efficiently. More precisely, the specific structure present in our context can be exploited, as we further describe in Section 5.3.

In our approach we consider a rank $N$ correction of the Sylvester operator, which can be expressed as a linear operator $\Pi$ of the form

$$
\Pi(X):=\sum_{k=1}^{N} \mathscr{W}_{k}(X) E_{k} .
$$

In this setting the matrix $W$ in (4.2) can be expressed in terms of evaluations of the functionals $\mathscr{W}_{1}, \ldots, \mathscr{W}_{N}$. This use of SMW is formalized in the following result.

Theorem 4.1 (Sylvester-type SMW-structure). Let $A \in \mathbb{C}^{n \times n}, B \in \mathbb{C}^{m \times m}$, and $C \in \mathbb{C}^{n \times m}$ and suppose $\operatorname{eig}(A) \cap \operatorname{eig}(-B)=\emptyset$. Moreover, let the matrices $E_{k} \in \mathbb{C}^{n \times m}$ and linear functionals $\mathscr{W}_{k}: \mathbb{C}^{n \times m} \rightarrow \mathbb{C}$ be given for $k=1,2, \ldots, N$ and define $\Pi: \mathbb{C}^{n \times m} \rightarrow \mathbb{C}^{n \times m}$ by (4.3). Assume that there exists a unique solution to the equation

$$
\mathscr{L}(X)+\Pi(X)=C
$$


where $\mathscr{L}$ is the Sylvester operator defined analogous to (3.2). Moreover, let

$$
\begin{aligned}
G & :=\mathscr{L}^{-1}(C), \quad \text { and } \\
F_{k} & :=\mathscr{L}^{-1}\left(E_{k}\right) \quad \text { for } k=1,2, \ldots, N .
\end{aligned}
$$

and define

$$
W:=\left(\begin{array}{cccc}
1+\mathscr{W}_{1}\left(F_{1}\right) & \mathscr{W}_{1}\left(F_{2}\right) & \ldots & \mathscr{W}_{1}\left(F_{N}\right) \\
\mathscr{W}_{2}\left(F_{1}\right) & 1+\mathscr{W}_{2}\left(F_{2}\right) & \ldots & \mathscr{W}_{2}\left(F_{N}\right) \\
\vdots & \vdots & \ddots & \vdots \\
\mathscr{W}_{N}\left(F_{1}\right) & \mathscr{W}_{N}\left(F_{2}\right) & \ldots & 1+\mathscr{W}_{N}\left(F_{N}\right)
\end{array}\right), g:=\left(\begin{array}{c}
\mathscr{W}_{1}(G) \\
\mathscr{W}_{2}(G) \\
\vdots \\
\mathscr{W}_{N}(G)
\end{array}\right)
$$

Then the solution to (4.4) is given by

$$
X=\mathscr{L}^{-1}\left(C-\sum_{k=1}^{N} \alpha_{k} E_{k}\right),
$$

where $a^{T}=\left(\alpha_{1}, \ldots, \alpha_{N}\right)$ is the unique solution to the system of equations

$$
W a=g .
$$

Proof. In order to invoke [8, Lemma 3.1] we note that the linear functionals $\mathscr{W}_{k}$, for $W_{k} \in \mathbb{C}^{n \times m}$, can be parametrized as $\mathscr{W}_{k}(X)=\operatorname{vec}\left(W_{k}\right)^{T} \operatorname{vec}(X)$. Moreover, we define the matrices $P_{1}:=\left[\operatorname{vec}\left(E_{1}\right), \ldots, \operatorname{vec}\left(E_{N}\right)\right]$, and $P_{2}:=\left[\operatorname{vec}\left(W_{1}\right), \ldots, \operatorname{vec}\left(W_{N}\right)\right]^{T}$. The conclusion (4.7)-(4.8) follows from direct reformulation of [8, Equation (6)].

REMARK 2 (Variants of Theorem 4.1). Note that, due to the linearity of $\mathscr{L}^{-1}$, the solution in (4.7) can be equivalently expressed as

$$
X=G-\sum_{k=1}^{N} \alpha_{k} F_{k}
$$

Moreover, since $G, F_{1}, \ldots, F_{N}$ can be treated as known, $X$ can be computed directly from (4.9) without the action of $\mathscr{L}^{-1}$. Hence, an approach based on (4.9) requires less computational effort than an approach based on (4.7) in general. However, in our case, (4.9) is not advantageous since it requires more memory resources as we further discuss in Section 5.2.

4.2. SMW-structure approximation of the waveguide matrix equation. We saw in the previous section that the matrix equation SMW-formula can be applied to sums of a Sylvester operator and the operator $\Pi$ in (4.3). Note that any linear matrix-operator can be expressed in the form (4.3), by selecting the functionals as $\mathscr{W}_{k}(X)=e_{j}^{T} X e_{\ell}$ and the matrices $E_{k}=e_{j} e_{\ell}^{T}$, where $j=1, \ldots, n$, and $\ell=1, \ldots, m$, and $k=j+(\ell-1) n$ such that $k \in\{1, \ldots, n m\}$ and $N=n m$. Unfortunately, such a construction is not practical since Theorem 4.1 is not computationally attractive for large values of $N$.

The equation (3.3) can be efficiently solved if the last terms in (3.3), i.e,

$$
\Phi(X):=\left(K-\bar{k} \mathbf{1} \mathbf{1}^{T}\right) \circ X-P_{-}(\sigma)^{-1} X E-P_{+}(\sigma)^{-1} X J E J,
$$

can be expressed as a low-rank operator $\Pi$ of the form (4.3). Then the solution to (3.3) can be directly computed with Theorem 4.1. In general, $\Phi$ can only be expressed 
as an operator $\Pi$ of large rank $N$. However, there exists some situations where exact matching can be achieved with $N \ll n_{x} n_{z}$, for instance if the elements of $K$ equals a constant, $\bar{k}$, except for a few indices. In the continuous formulation, this corresponds to the wavenumber being constant in most parts of the domain.

Although, $\Phi$ can in general only be expressed in the form of (4.3) with a large $N$, we now introduce a low-rank approximation of $\Phi$, i.e., with $N \ll n_{x} n_{z}$. Our construction exploits the structure in $\Phi$ and allows for a representation of $\Pi$ with both low rank $N$ and structured matrices $E_{k}$.

We consider approximations in a vector space $\mathcal{V} \subset \mathbb{C}^{n_{z} \times n_{x}}$, with a basis $V_{1}, \ldots, V_{N}$, which is assumed to be orthogonal with respect to the trace inner product $\langle X, Y\rangle=$ $\operatorname{Tr}\left(Y^{*} X\right)$. We take approximation of $X \in \mathbb{C}^{n_{z} \times n_{x}}$ from this space and let $\tilde{X}$ be the best approximation (in the induced trace norm). Equivalently we can impose the Galerkin condition on $X$,

$$
\left\langle X-\tilde{X}, V_{k}\right\rangle=0, \quad \text { for } k=1, \ldots, N,
$$

which leads to the formula $\tilde{X}:=\sum_{k=1}^{N} \frac{\left\langle X, V_{k}\right\rangle}{\left\langle V_{k}, V_{k}\right\rangle} V_{k}$. Based on this approximation, we construct $\Pi$ as an approximation of $\Phi$ by setting

$$
\Pi(X):=\Phi\left(\sum_{k=1}^{N} \frac{\left\langle X, V_{k}\right\rangle}{\left\langle V_{k}, V_{k}\right\rangle} V_{k}\right)=\sum_{k=1}^{N} \frac{\left\langle X, V_{k}\right\rangle}{\left\langle V_{k}, V_{k}\right\rangle} \Phi\left(V_{k}\right) .
$$

If we define $\mathscr{W}_{k}(X):=\frac{\left\langle X, V_{k}\right\rangle}{\left\langle V_{k}, V_{k}\right\rangle}$ and $E_{k}:=\Phi\left(V_{k}\right)$, then $\Pi$ is of the form (4.3). More precisely, for our structure in (4.10) we have

$$
E_{k}:=\left(K-\bar{k} \mathbf{1} \mathbf{1}^{T}\right) \circ V_{k}-P_{-}(\sigma)^{-1} V_{k} E-P_{+}(\sigma)^{-1} V_{k} J E J .
$$

As can be expected from a Galerkin approach, the approximation is exact for any $X \in \mathcal{V}$, since by construction $\Phi\left(V_{k}\right)=\Pi\left(V_{k}\right), k=1, \ldots, N$.

In theory, the construction can be done for any appropriate vector space. For reasons of structure exploitation, we select $V_{k}, k=1, \ldots, N$, as indicator functions in rectangular regions, as shown in Figure 4.1. We then select $N_{x}$ and $N_{z}$ intervals in $x$ and $z$-direction respectively, hence $N=N_{x} N_{z}$. In this case the matrices $V_{\ell+m\left(N_{z}-1\right)}$, $\ell=1, \ldots, N_{z}$ and $m=1, \ldots, N_{x}$, take the value 1 in the corresponding rectangular region and zero outside, and $\mathscr{W}_{\ell+m\left(N_{z}-1\right)}$ is the functional taking the mean over that region. More precisely,

$$
\begin{aligned}
{\left[V_{\ell+m\left(N_{z}-1\right)}\right]_{p, q} } & = \begin{cases}1 & \text { if }(p, q) \text { belongs to the region }(\ell, m) \\
0 & \text { otherwise }\end{cases} \\
\mathscr{W}_{\ell+m\left(N_{z}-1\right)}(X) & =\frac{\sum_{p, q}\left[X \circ\left[V_{\ell+m\left(N_{z}-1\right)}\right]\right]_{p, q}}{\sum_{p, q}\left[V_{\ell+m\left(N_{z}-1\right)}\right]_{p, q}} .
\end{aligned}
$$

Note that the grid resolution is finer near the boundary. This is done in order to improve the approximation of the DtN-maps, which are localized in the boundary region. The localization can be seen in the structure of the E-matrix in Proposition 3.1. This choice is supported by computational experiments presented in Section 6 .

REMARK 3 (Other approximations). Note that the approximation described above is only an illustration of an approximation procedure and there exist many variations. Apart from other Galerkin type approaches, it is also possible to use the rank-revealing 
procedure proposed in [8, Algorithm 3.2]. Another option is to use smoothing as, e.g., in multi-grid methods and other domain decomposition methods [10]. In order to use these approaches in the framework here described, further focused research would be required. In our setting, the structure of (4.11) allows us to reduce the memory requirements as we describe in the next section.

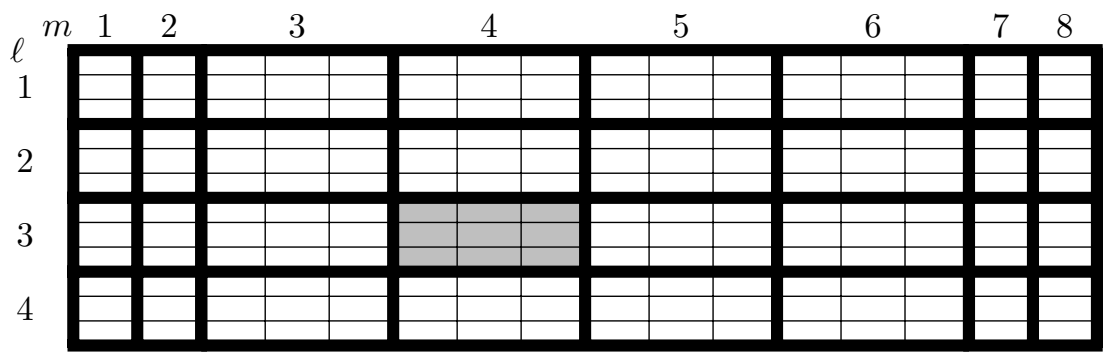

FIGURE 4.1. The coarse grid shows the regions in which we take basis vectors $V_{k}$ as constant. The fine grid is an illustration of the underlying grid from the discretization of the PDE. For example the weight matrix $V_{3+4\left(N_{z}-1\right)}$ will have value 1 in the light gray area and 0 on all other elements. Note that the coarse grid for the SMW approximation (SMW-grid) is finer towards the boundaries in $x$-direction, this is to better capture the effect of the DtN-maps.

\section{Structure exploitation and specialization of Resinv.}

5.1. SMW-preconditioned Resinv. The application of Resinv to the WEP, described in Section 2.3, requires an efficient solution to the linear system $M(\sigma)^{-1} r$ in equation (2.12). Since we want to solve this linear system iteratively, we need an effective preconditioner. We use the approximation technique presented in Section 4.2 as a preconditioner. As a consequence of the fact that the shift is kept constant in Resinv, the Sylvester operator defined in (3.2), with $A:=D_{z z}+2 \sigma D_{z}+\sigma^{2} I_{n_{z}}+\bar{k} I_{n_{z}}$ and $B:=D_{x x}$, is also constant. Therefore the matrices $F_{1}, \ldots, F_{N}$ in $(4.5 \mathrm{~b})$ and the $W$-matrix in (4.6) are constant over the iterations. Hence, the $W$-matrix can be precomputed before initiating Resinv. Moreover, as mentioned in Remark 2, this formulation of the SMW-formula, does not require the storage of the matrices $F_{1}, \ldots, F_{N}$, once $W$ has been computed. In fact only one $F$-matrix needs to be stored at a time, since the columns of $W$ can be computed column-wise. The precomputation can also be trivially parallelized since the columns of $W$ are independent. This construction is summarized in Algorithm 2.

An important feature of the algorithm is that $N$ can be treated as a parameter. Using a large $N$ implies more computational work in the precomputation phase, i.e., step 1-4 of Algorithm 2, since many Sylvester equations need to be solved. However, the quality of the preconditioner is better and we expect the iterative method to convergence in fewer iterations for large $N$. More precisely, less computation is required for the iterative solves ${ }^{1}$ in Step 9 . Hence, $N$ parameterizes a trade-off between computation time in the initialization and in the iterative solves. As is illustrated in Section 6 , the best choice of $N$ in terms of total computation time is a non-trivial problem.

In order to further improve performance we use a result regarding residual inverse iteration in [33]. More precisely, [33, Theorem 9] states that the linear solves in Resinv can be terminated in a way that preserves the property that the convergence factor

\footnotetext{
${ }^{1}$ This holds only if $N \ll n_{x} n_{z}$ as computation of (4.8) otherwise is a significant part of Step 9.
} 
is proportional to the shift-eigenvalue distance. It is proposed to use the tolerance $\tau$ satisfying

$$
\left\|M\left(\gamma^{k+1}\right) v_{k}-M(\sigma) \Delta v_{k}\right\| \leq \tau\left\|M\left(\gamma^{k+1}\right) v_{k}\right\|
$$

and $\tau=\mathcal{O}\left(\left|\gamma_{*}-\sigma\right|\right)$. Although, we solve the linear system (2.15) inexactly, the error propagates linearly to $(2.14)$, and hence the tolerance (5.1) is natural also in our setting.

5.2. Storage improvements. The particular choice of SMW-formulation is due to an observation in computational experiments, that memory is a restricting aspect in our approach. We have therefore selected the SMW-formulation in order to reduce memory requirement at the cost of an increased computation time. Our approach requires the computation of the solution to two Sylvester equations per iteration (equation (4.5a) and (4.7)). The solution $X$ in (4.7) could be computed by forming a linear combination of $G, F_{1}, \ldots, F_{N}$, as in (4.9) but would require the storage of $F_{1}, \ldots, F_{N}$ which are full matrices in general. In contrast to this, the matrices $E_{1}, \ldots, E_{N}$ have a structure that can be exploited. More precisely, the matrix $C-\sum_{k=1}^{N} \alpha_{k} E_{k}$ required in (4.7) can be computed efficiently by exploiting the structure of $V_{k}$ and $E_{k}$ defined in (4.11).

5.3. Circulant structure exploitation for Sylvester equation. In order to use the suggested preconditioner we need to solve a number of Sylvester equations, with the Sylveser operator defined by (3.2). There are many methods available in the literature, both direct methods such as the Bartels-Steward algorithm [2] as well as iterative methods. See [30] for a recent survey of available methods. These are general methods for solving the Sylvester equation. However, our Sylvester equation has a particular structure which can be exploited further. The approach is based on the implicit diagonalization of the coefficient matrices, from which a closed form expression is available [30]. Consider the equation $A X+X B=C$ where $A$ and $B$ are diagonalizable. Then the solution $X$ is given by

$$
X=V Y W^{-1}, \quad \text { with } \quad[Y]_{p, q}=\frac{\left[V^{-1} C W\right]_{p, q}}{\left[\Lambda_{A}\right]_{p}+\left[\Lambda_{B}\right]_{q}},
$$

where $A=V \Lambda_{A} V^{-1}$, and $B=W \Lambda_{B} W^{-1}$. In the general case, the application of (5.2) is expected to be expensive and numerically unstable. For the waveguide matrix equation (3.3) the matrix $A$ is circulant, as it stems from the discretization with periodic boundary conditions. In particular it is diagonalized by the Fourier matrix [11, Theorem 4.8.2] whose action can be computed by FFT; the eigenvalues are also readily available in $\mathcal{O}\left(n_{z} \log \left(n_{z}\right)\right)$ operations using FFT [11]. The other matrix $B=D_{x x}$ is well studied and has both known eigenvalues and eigenvectors, the action of the latter can be computed in an efficient and stable way using the relation between Sine-/Cosine-transforms and FFT [9, Lemma 6.1] [11, Section 4.8]. The solution to the Sylvester operator in (3.2), i.e.,

$$
\left(D_{z z}+2 \sigma D_{z}+\left(\sigma^{2}+\bar{k}\right) I_{n_{z}}\right) X+X D_{x x}=C,
$$

can hence be computed by using (5.2) since the action of $V, V^{-1}, W$, and $W^{-1}$ can be computed efficiently and accurately using FFT, and the diagonals of $\Lambda_{A}$ and $\Lambda_{B}$ are available. This exploitation of FFT leads to a computation complexity $\mathcal{O}\left(n_{x} n_{z} \log \left(n_{x} n_{z}\right)\right)$ for the inversion of (5.3), cf. [9, Section 6.7]. 


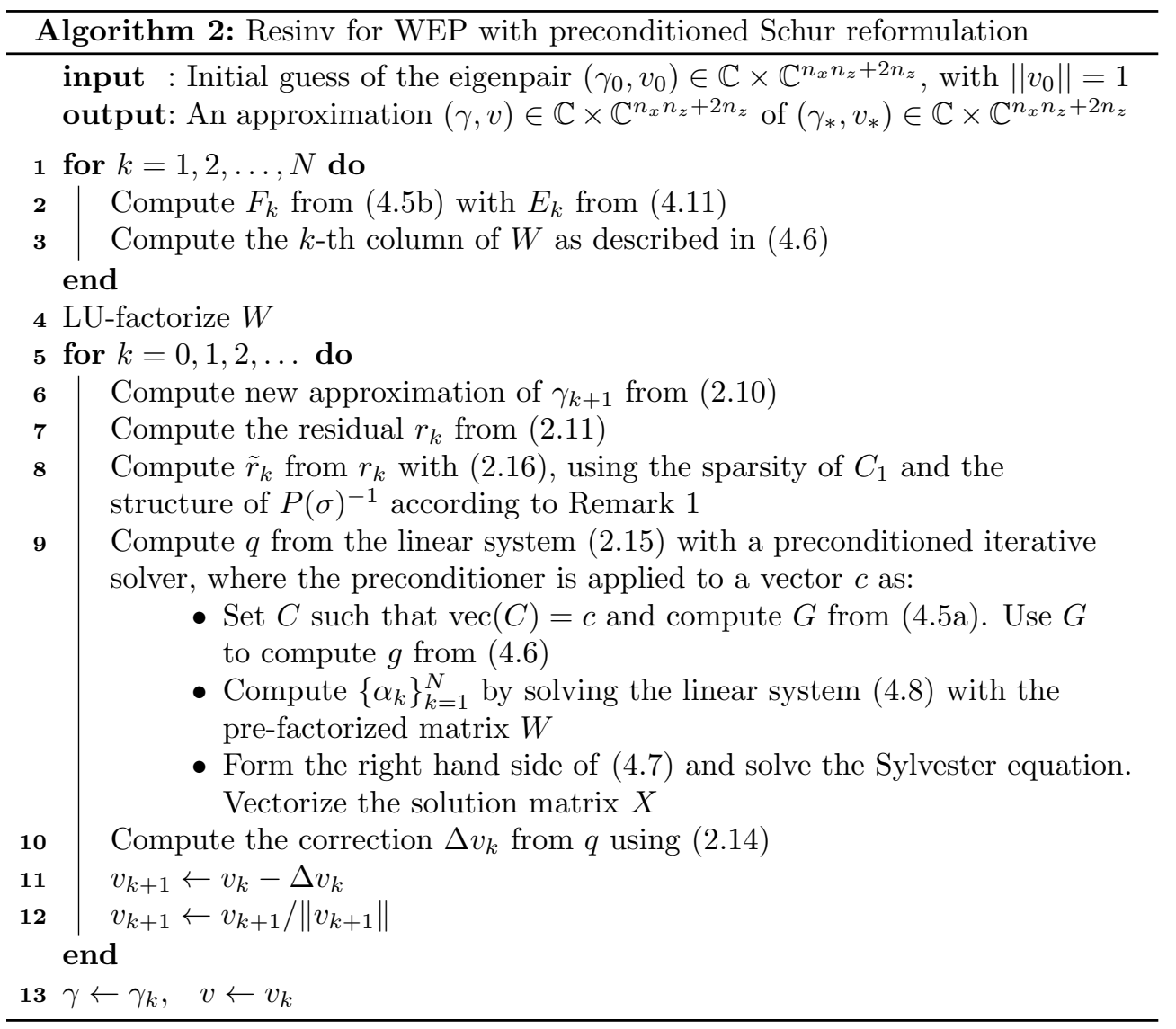

6. Numerical simulations. In order to illustrate properties of our approach, we now show the result of simulations carried out in MATLAB on a desktop computer. ${ }^{2}$ Source code for the simulations are provided online to improve reproducability. ${ }^{3} \mathrm{We}$ use the waveguide illustrated in Figure 1.1a, and also described in [15, Section 5.2]. This waveguide has many eigenvalues, oscillatory eigenfunctions, and large discontinuity in the wavenumber, which is constructed to be representative of a realistic situation. In the simulations we set the free parameter $\bar{k}$, as introduced in the waveguide matrix equation (3.3), to $\bar{k}=\operatorname{mean}(K)$. Moreover, the size of the problem is denoted $n$ and defined as $n:=n_{x} n_{z}+2 n_{z}$, and the parametrization of the preconditioner, $N$, is defined by $N:=N_{x} N_{z}$. For implementation convenience we select $n_{x}=n_{z}+4$ and $N_{x}=N_{z}+4$.

We first illustrate the quality of the preconditioner (without incorporation into Resinv). The relative error as function of GMRES-iteration is visualized in Figure 6.1. We clearly see that the required number of iterations decreases with $N$, which is expected since the SMW-approximation error is smaller for larger $N$. Moreover, we see that a small $N$ normally generates a long transient phase. The advantage of selecting a finer grid close to the boundary as shown in Figure 4.1 is clear from the

\footnotetext{
${ }^{2}$ Intel quad-core i5-5250U CPU $1.60 \mathrm{GHz} \times 4$, with 16 GB RAM using MATLAB 2015a (8.5.0.197613).

${ }^{3}$ URL: https://www.math.kth.se/ eringh/software/wep/wep_code
} 
fact that the convergence in Figure 6.1a is faster than the convergence in Figure 6.1b.

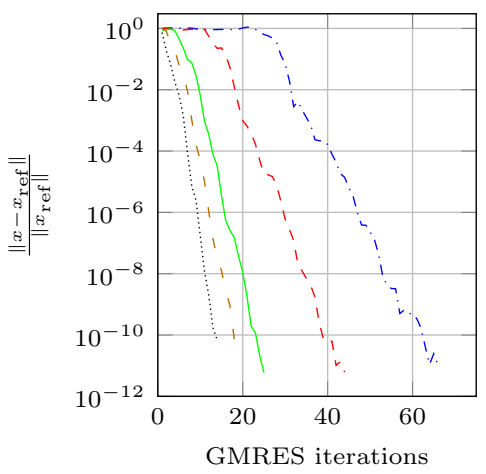

(a) With the SMW-grid from Figure 4.1, where $N_{x}=N_{z}+4$.

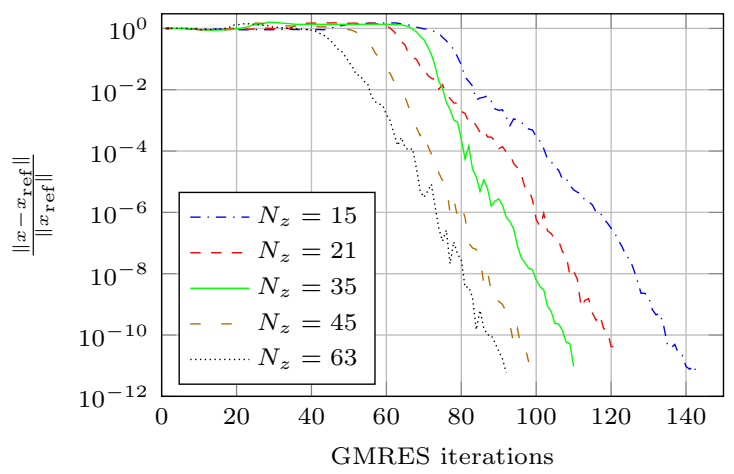

(b) With a uniform coarser grid (cf. Figure 4.1). Note that here $n_{x}=n_{z}$, and $N_{x}=N_{z}$.

FIGURE 6.1. GMRES convergence for solving $S(\sigma) x=c$ for different coarse grids in the $S M W$-approximation. The discretization is $n_{z}=945$ and the error is measured as the relative error compared to a reference solution $x_{r e f}$.

For the remaining simulations we measure the error of the approximation with an estimate of the relative residual norm

$$
R(v, \gamma)=\frac{\|M(\gamma) v\|_{2}}{\sum_{k=0}^{2}|\gamma|^{k}\left\|A_{k}\right\|_{1}+\left\|C_{1}\right\|_{1}+\left\|C_{2}^{T}\right\|_{1}+2\left|d_{0}\right|+\sum_{k=-p}^{p}\left(\left|s_{+, k}(\gamma)\right|+\left|s_{-, k}(\gamma)\right|\right)}
$$

analogous to estimates for other NEPs [20, 13]. Algorithm 2 is applied to this benchmark problem, and the error is visualized in Figure 6.2a. We use $\sigma=-0.5-0.4 i$, such that the algorithm converges to the eigenvalue $\gamma \approx-0.523-0.375 i$. As expected from the GMRES-termination criteria (5.1), we maintain linear convergence and a convergence factor which is in the order of magnitude of the shift-eigenvalue distance. The corresponding computed eigenfunction is visualized in Figure 6.2b.

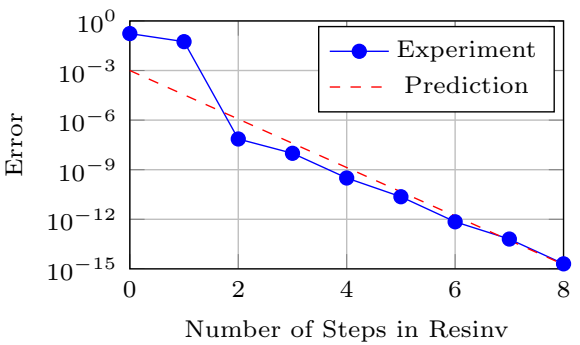

(a) Error in relative residual norm (6.1). The prediction corresponds to convergence factor $|\gamma-\sigma|$

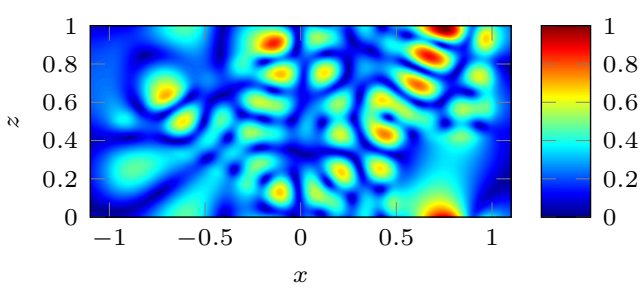

(b) Absolute value of the eigenfunction.

FIGURE 6.2. Illustration of convergence of Resinv and the corresponding eigenfunction to eigenvalue $\gamma \approx-0.523-0.375 i$. In this simulation we use the shift $\sigma=-0.5-0.4 i$, the discretization $n_{z}=2835$, and the preconditioner parameter $N_{z}=21$.

Figure 6.3 shows the number of required GMRES-iterations for one iteration of Resinv. As expected from the approximation properties of the preconditioner, a larger 
value $N$ implies fewer iterations. We observe an increase in the number of required GMRES-iterations with increasing problem size. The increase is however rather slow.

The choice of $N$ for parameterizing the trade-off between precomputation time and times in the solves, pointed out in Section 5.1, is illustrated in terms computationtime in Figure 6.4a. For this particular problem (and computing environment) the best choice is $N_{z}=35$. Note however, that several other choices such as $N_{z}=45$ and $N_{z}=63$ are almost as good. In profiling illustration in Figure $6.4 \mathrm{~b}$, we see, as expected, that increasing $N$, shifts computational effort into to the precalculation phase, and that the computational effort required for the precalculation and the other parts of the algorithm are of the same order of magnitude.

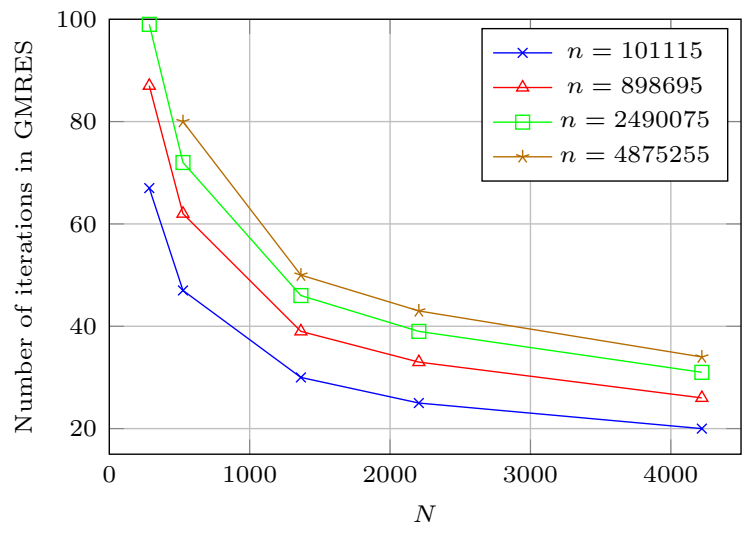

FiguRE 6.3. Illustration of the increase in GMRES iterations. Evaluations for SMW-grid parameters $N_{x}=N_{z}+4$ and $N_{z} \in\{15,21,35,45,63\}$ where $N=N_{x} N_{z}$.

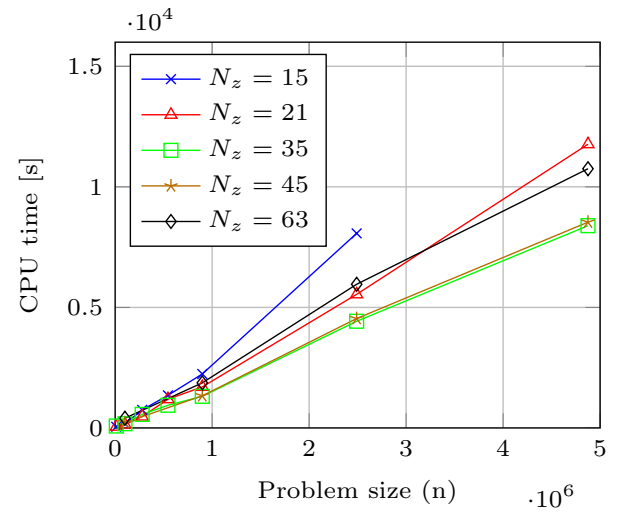

(a) Time in seconds for solving the problem.

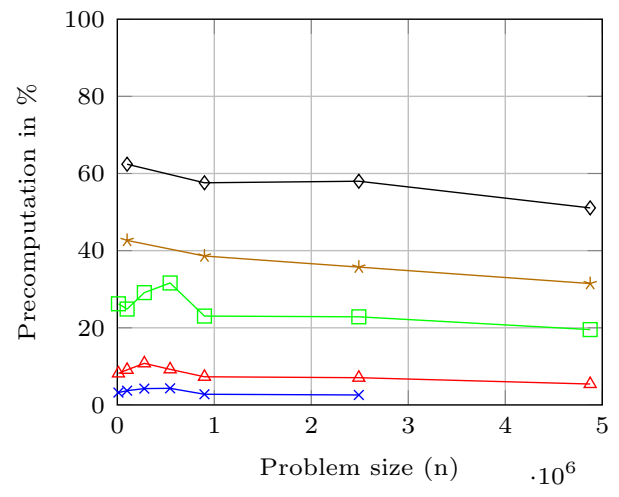

(b) Percentage of time spent on precalculating the (SMW) $W$-matrix from (4.6).

Figure 6.4. Time for the complete method described in Algorithm 2, as a function of problem size. Problem size is the total size of the problem as presented in (1.2), that is $n_{x} n_{z}+2 n_{z}$, where the GMRES-tolerance is select such that linear system is solved to full precision. This is plotted for different $N$ values $N=N_{x} N_{z}$, and $N_{x}=N_{z}+4$.

We now illustrate our experiences with the capabilities of the full approach. We carry out simulations with the iterative methods GMRES and BiCGstab using the 
proposed preconditioner. In addition to this we compare with GMRES combined with a preconditioner based on incomplete LU-factorization (ILU) [27, Chapter 10]. The computation times are given in Table 6.1. For very large problems, GMRES is not advantageous due to memory requirements. Dealing with this using restarted GMRES is not competitive due to long transient phases, similar to those observed in Figure 6.1. GMRES requires more memory than BiCGStab, and the largest problem we manage to solve is computed with BiCGstab. ${ }^{4}$ However, GMRES was in general slightly faster in simulations with enough memory. For large problems with GMRES, the size of the Krylov space needs to be carefully adjusted to stay within the available RAM. For most simulations the maximum size of the Krylov space is 100 vectors, but for $n \approx 16 \cdot 10^{6}$ only 30 vectors is used for $N_{z}=35$ and $N_{z}=21$. However, for the case $N_{z}=15$ no size of the Krylov subspace is found that is sufficiently large to reach convergence, but small enough to stay within the available RAM. Unfortunately, in our experiments ILU required considerable memory resources and we were not able to use it to solve very large problems.

A FEM-discretization of this problem was presented in [15]. Our preconditioner can also be applied to solve the discretization with FEM, by using the FDpreconditioner. The observed convergence is similar to the previously observed convergence in the FD-case (cf. Figure 6.1a). For instance, when our method is applied to a discretization with $n_{z}=945$ and $N_{z}=21$, GMRES for the FD-problem needs 44 iterations and GMRES for the FEM-problem need 44 iterations as well.

\begin{tabular}{r||c|c|c||c|c|c||c|c}
\multicolumn{1}{c||}{} & \multicolumn{4}{c||}{ GMRES } & \multicolumn{3}{c||}{ BiCGStab } & ILU (GMRES) \\
\hline$n$ & 15 & 21 & 35 & 15 & 21 & 35 & $10^{-5}$ & $10^{-6}$ \\
\hline \hline 101115 & 63 & 53 & 79 & 85 & 66 & 91 & 78 & 74 \\
278775 & 201 & 169 & 241 & 324 & 216 & 246 & 504 & 474 \\
898695 & 542 & 405 & 509 & 757 & 520 & 570 & $\star$ & $\star$ \\
2490075 & 1674 & 1129 & 1652 & 2391 & 1668 & 1796 & $\star$ & $\star$ \\
4875255 & 3530 & 2435 & 3218 & 4653 & 3199 & 3276 & $\star$ & $\star$ \\
8054235 & 6732 & 4675 & 6658 & 10808 & 7001 & 7528 & $\star$ & $\star$ \\
16793595 & $\star$ & 11171 & 17116 & 23047 & 14865 & 15983 & $\star$ & $\star$
\end{tabular}

Illustration of CPU-time in seconds for a set of different methods. The preconditioner parameter $p$ is $p=N_{z}$ for the proposed preconditioner, $N=N_{x} N_{z}$ where $N_{x}=N_{z}+4$; and for ILU $p=\epsilon$ the dropping tolerance. The symbol $\star$ denotes simulations which could not be executed due to insufficient $R A M$.

7. Concluding remarks and outlook. We have presented a new computational procedure specialized for the WEP (1.1), based on combining the method for NEPs called Resinv and an iterative method for linear systems. The preconditioner for the iterative method is based on an approximation leading to a structure which can be exploited with a matrix-equation version of SMW.

There are many options for constructing the SMW approximation. For instance, the space used in the Galerkin approximation could be selected in a number of ways. Such a construction would necessarily need to use sparsity or other matrix structures in order to solve large-scale problems.

\footnotetext{
${ }^{4}$ This size is larger than $28 \cdot 10^{6} \times 28 \cdot 10^{6}$.
} 
We have focused on one particular method for NEPs: Resinv. One of the crucial features is that a linear system corresponding to $M(\sigma)$ needs to be solved many times (for a constant shift). The Resinv method is not the only method that uses the solution to many linear systems with a fixed shift. This is also the case for the nonlinear Arnoldi method [36] and the tensor infinite Arnoldi method [15]. However, inexact solves in Arnoldi-type methods are sometimes problematic [31], and further research would be required in order to reliably and efficiently use our preconditioner for these methods.

Although our approximation is justified with a Galerkin approach, we have not provided any theoretical convergence analysis. The application of standard prooftechniques for such an analysis, e.g., involving eigenvalues and spectral condition numbers have not lead to a clear characterization of the error. Therefore, we believe that a convergence analysis would require use of the regularity of the eigenfunction, similiar to what is used in multi-grid methods [10], which is certainly beyond the scope of this paper.

Finally, we wish to point out that several results in this paper may be of interest also for other problems and other NEPs. Most importantly, many NEPs arise naturally from artificial boundary conditions. Most artificial boundary conditions has freedom regarding selection of boundary. We can therefore select a rectangular domain, i.e., similar to the framework considered in this paper.

Acknowledgment. The authors wish to thank Per Enqvist (KTH) and Tobias Damm (TU Kaiserslautern) for comments and discussions in the early developments of this result. This research is supported by the Swedish research council under Grant No. 621-2013-4640.

\section{REFERENCES}

[1] J. Asakura, T. Sakurai, H. Tadano, T. Ikegami, and K. Kimura. A numerical method for nonlinear eigenvalue problems using contour integrals. JSIAM Letters, 1:52-55, 2009.

[2] R. Bartels and G. W. Stewart. Solution of the matrix equation $A X+X B=C$. Comm A.C.M., 15(9):820-826, 1972

[3] R. Van Beeumen, K. Meerbergen, and W. Michiels. A rational Krylov method based on Hermite interpolation for nonlinear eigenvalue problems. SIAM J. Sci. Comput., 35(1):A327-A350, 2013.

[4] R. Van Beeumen, K. Meerbergen, and W. Michiels. Compact rational Krylov methods for nonlinear eigenvalue problems. SIAM J. Sci. Comput., 36(2):820-838, 2015.

[5] T. Betcke, N. J. Higham, V. Mehrmann, C. Schröder, and F. Tisseur. NLEVP: A collection of nonlinear eigenvalue problems. ACM Trans. Math. Softw., 39(2):1-28, 2013.

[6] W.-J. Beyn. An integral method for solving nonlinear eigenvalue problems. Linear Algebra Appl., 436(10):3839-3863, 2012.

[7] T. Breiten, V. Simoncini, and M. Stoll. Low-rank solvers for fractional differential equations. Electronic Transactions on Numerical Analysis, 45:107-132, 2016.

[8] T. Damm. Direct methods and ADI-preconditioned Krylov subspace methods for generalized Lyapunov equations. Numer. Linear Algebra Appl., 15:853-871, 2008.

[9] J. Demmel. Applied numerical linear algebra. SIAM publications, Philadelphia, PA, 1997.

[10] V. Dolean, P. Jolivet, and F. Nataf. An Introduction to Domain Decomposition Methods. SIAM publications, Philadelphia, PA, 2015.

[11] G. Golub and C. Van Loan. Matrix Computations. The Johns Hopkins Univ. Press, Baltimore, MD, 4th edition, 2013.

[12] S. Güttel, R. Van Beeumen, K. Meerbergen, and W. Michiels. NLEIGS: a class of fully rational Krylov methods for nonlinear eigenvalue problems. SIAM J. Sci. Comput., 36(6):A2842A2864, 2014.

[13] N. J. Higham, R.-C. Li, and F. Tisseur. Backward error of polynomial eigenproblems solved by linearization. SIAM J. Matrix Anal. Appl., 29(4):1218-1241, 2008. 
[14] R. Horn and C. Johnson. Topics in Matrix Analysis. Cambridge Univ. Press, Cambridge, UK, 1991.

[15] E. Jarlebring, G. Mele, and O. Runborg. The waveguide eigenvalue problem and the tensor infinite Arnoldi method. Technical report, KTH Royal Institute of Technology, 2015. arxiv preprint.

[16] E. Jarlebring and W. Michiels. Analyzing the convergence factor of residual inverse iteration. $B I T, 51(4): 937-957,2011$.

[17] E. Jarlebring, W. Michiels, and K. Meerbergen. A linear eigenvalue algorithm for the nonlinear eigenvalue problem. Numer. Math., 122(1):169-195, 2012.

[18] D. Kressner. A block Newton method for nonlinear eigenvalue problems. Numer. Math., 114(2):355-372, 2009.

[19] I. Kuzmanovi and N. Truhar. Sherman-Morrison-Woodbury formula for Sylvester and TSylvester equations with applications. Int. J. Comput. Math., 90(2):306-324, 2013.

[20] B.-S. Liao, Z. Bai, L.-Q. Lee, and K. Ko. Solving large scale nonlinear eigenvalue problems in next-generation accelerator design. Technical Report SLAC-PUB-12137, Stanford University, 2006.

[21] V. Mehrmann and H. Voss. Nonlinear eigenvalue problems: A challenge for modern eigenvalue methods. GAMM-Mitt., 27:121-152, 2004.

[22] A. Neumaier. Residual inverse iteration for the nonlinear eigenvalue problem. SIAM J. Numer. Anal., 22:914-923, 1985.

[23] D. Palitta and V. Simoncini. Matrix-equation-based strategies for convection-diffusion equations. BIT, pages $1-26,2015$.

[24] C. E. Powell, D. Silvester, and V. Simoncini. An efficient reduced basis solver for stochastic Galerkin matrix equations. Technical report, Univ. Manchester, 2015. MIMS EPrint 2015.64 .

[25] S. Richter, L. D. Davis, and E. G. Collins Jr. Efficient computation of the solutions to modified Lyapunov equations. SIAM J. Matrix Anal. Appl., 14(2):420-431, 1993.

[26] A. Ruhe. Algorithms for the nonlinear eigenvalue problem. SIAM J. Numer. Anal., 10:674-689, 1973.

[27] Y. Saad. Iterative methods for sparse linear systems. SIAM publication, Philadelphia, PA, 1996.

[28] Y. Saad and M. H. Schultz. GMRES: A generalized minimal residual algorithm for solving nonsymmetric linear systems. SIAM J. Sci. Stat. Comput., 7:856-869, 1986.

[29] K. Schreiber. Nonlinear Eigenvalue Problems: Newton-type Methods and Nonlinear Rayleigh Functionals. PhD thesis, TU Berlin, 2008.

[30] V. Simoncini. Computational methods for linear matrix equations. SIAM Review, 58(3):377$441,2016$.

[31] V. Simoncini and D. B. Szyld. Theory of inexact Krylov subspace methods and applications to scientific computing. SIAM J. Sci. Comput., 25(2):454-477, 2003.

[32] M. Stoll and T. Breiten. A low-rank in time approach to PDE-constrained optimization. SIAM J. Sci. Comput., 37(1):B1-B29, jan 2015.

[33] D. B. Szyld and F. Xue. Local convergence analysis of several inexact Newton-type algorithms for general nonlinear eigenvalue problems. Numer. Math., 123(2):333-362, 2012.

[34] J. Tausch and J. Butler. Floquet multipliers of periodic waveguides via Dirichlet-to-Neumann maps. J. Comput. Phys., 159(1):90-102, 2000.

[35] H. A. van der Vorst. BI-CGSTAB: A fast and smoothly converging variant of BI-CG for the solution of nonsymmetric linear systems. SIAM J. Sci. and Stat. Comput., 13(2):631-644, 1992.

[36] H. Voss. An Arnoldi method for nonlinear eigenvalue problems. BIT, 44:387 - 401, 2004.

[37] H. Voss. Nonlinear eigenvalue problems. In L. Hogben, editor, Handbook of Linear Algebra, Second Edition, number 164 in Discrete Mathematics and Its Applications. Chapman and Hall/CRC, 2013.

[38] J. Xiao, C. Zhang, T.-M. Huang, and T. Sakurai. Solving large-scale nonlinear eigenvalue problems by rational interpolation and resolvent sampling based Rayleigh-Ritz method. Internat. J. Numer. Methods Engrg, 2016. 